\title{
DER UMLAUF DER SPANISCHEN HANDSCHRIFTEN AUS DEM ANTIQUARIAT LEO LIEPMANNSSOHNS IN PARIS UND BERLIN ${ }^{1}$
}

\author{
Francisco Javier ROMERO NARANJO
}

\begin{abstract}
One of the antique libraries most representative of the second half of the $19^{\text {th }}$ century was that of Leo Liepmannssohn, with branches in Paris and Berlin. Its importance grew over time due to its auctions of many manuscripts by J. S. Bach, Handel, Mozart, Beethoven and other composers, a fact that explains how the author came across it during his investigation into the referred manuscripts. The object of this investigation is a collection of more than 300 folders that can be consulted in the Music Department in the Staatsbibliothek Preußischer Kulturbesitz in Berlin. The folders contain the annual catalogues from the antique library of Liepmannssohn (Berlin) comprising the years 1866 to 1923. Each catalogue consists of a list of all the sales from the corresponding year in alphabetical order and contains, besides manuscripts, literature, musical treatises and the portraits of several composers. There are many Spanish manuscripts included in these catalogues whose distribution was so important that in 1900 Liepmannssohn published an exclusive catalogue for Spanish and Portuguese manuscripts, 291 of which the librarian had sold during that year. During the celebration of those auctions many documents, musical treatises and manuscripts, both known and unknown, were brought to light. The investigation carried out contributes to our knowledge about the works of different Spanish composers from the $16^{\text {th }}$ to the $19^{\text {th }}$ centuries and their distribution abroad, especially in Berlin.
\end{abstract}

\section{Zusammenfassung}

Eines der repräsentativsten Antiquariate der zweiten Hälfte des XIX Jh. und des Anfangs des XX Jh. wurde von Leo Liepmannssohn in den Städten Paris und Berlin verwaltet. Durch die Versteigerung vieler Handschriften von J.S. Bach, Händel, Mozart, Beethoven und anderer Komponisten gewann das Antiquariat zusätzlich an Bedeutung. Deswegen ist es verständlich, dass der Autor auf das Antiquariat stieß, als er Nachforschungen über den Umlauf dieser Handschriften anstellte. Der Forschungsgegenstand beinhaltet eine Sammlung von mehr als 300 Heften, die in der Musikabteilung der Staatsbibliothek Preußischer Kulturbesitz zu Berlin einzusehen sind. Die Hefte umfassen die Jahreskataloge des Liepmannssohnschen Antiquariates (Berlin) der Jahre 1866 bis 1923. Jeder Katalog hat alle Verkäufe des jeweiligen Jahres in alphabetischer Reihenfolge aufgelistet und enthält neben Handschriften auch Literatur, Musikabhandlungen und Portraits verschiedener Komponisten. In diesen Katalogen sind sehr viele spanische Handschriften aufgeführt. Der Umlauf all dieser Handschriften ist so wichtig, dass Leo Liepmannssohn 1900 einen Katalog nur mit spanischen und portugiesischen Handschriften veröffentlicht hat. In jenem Jahr hatte Liepmannssohn 291 spanische und portugiesische Handschriften versteigert. In diesen Versteigerungen tauchten bekannte - aber auch viele unbekannte - Dokumente, Handschriften und Musikabhandlungen auf. Durch diese Forschung wurden die Kenntnisse über Kompositionen verschiedener spanischer Komponisten des XVI. bis XIX. Jh. erweitert und im Ausland - speziell in Berlin verbreitet.

1. Auf Basis dieses Artikels wurde 2002 auf dem 17. Kongress in der Internationalen Gesellschaft für Musikwissenschaft ein Vortrag gehalten.

*. Herzlichen Dank an die Familie Reinhardt, besonders an Felix Reinhardt für die Hilfe.

Anuario Musical, 59 (2004) 
Der Einfluss und Aufenthalt ausländischer Musiker in Spanien wurde vermehrt im letzten Viertel des 20. Jahrhunderts erforscht, die Strömung und Rezeption spanischer Komponisten jenseits der spanischen Grenzen hingegen wurde in geringerem Maße behandelt.

Dennoch haben einige Autoren einer Anzahl Komponisten, die im Ausland durch ihr Talent glänzten, besondere Beachtung geschenkt. An dieser Stelle könnte man eine ganze Reihe von Dokumenten nennen zu Komponisten wie die Gebrüder Plá ${ }^{2}$, Juan Oliver Astorga ${ }^{3}$, Vicente Martín y Soler ${ }^{4}$, etc.

Die gegenwärtige Kenntnis über das Thema ist den Dokumenten zu verdanken, die in verschiedenen europäischen Archiven gefunden wurden. Darunter eine Reihe autographer Handschriften oder Kopien, Musikalmanache, Reiseberichte etc., die auf direkte und indirekte Weise zahlreiche Informationen liefern.

Bis zum gegenwärtigen Zeitpunkt hat man Kenntnis vom zeitlich begrenzten Aufenthalt vieler spanischer Komponisten im Ausland und auch von jenen, die ihr ganzes Leben jenseits der spanischen Grenzen verbrachten, obschon sie spanischer Abstammung waren (wie beispielsweise Carlos Ordóñez oder Marianna Martines). Dennoch findet man auch eine große Anzahl von Werken spanischer Komponisten, die nicht im Ausland waren, deren Ruhm aber weit über die spanischen Grenzen hinaus reichte.

Ihre Werke gelangten auf verschiedenen Wegen nach Frankreich, Italien, Portugal und Deutschland. Einerseits waren die Kopien von großer Bedeutung (siehe die Wichtigkeit der wienerischen Kopisten während des 18. Jahrhunderts), anderseits der Kauf publizierter Werke oder der Erwerb durch Antiquarier, die anschliessend an öffentliche Anstalten (wie staatliche Bibliotheken) oder private Käufer verkauften.

Die Antiquitätenhändler haben beim Umlauf von Handschriften eine besondere Rolle gespielt, weil sie eine große Menge von Werken verschiedener Autoren unterschiedlicher Länder sammelten, die sie später versteigerten. In diesem Zusammenhang muss auch die Bedeutung des Antiquars Leo Liepmannssohn für die Städte Paris und Berlin hervorgehoben werden ${ }^{5}$.

Das Hauptziel dieses Artikels besteht darin, einen Beitrag zur Kenntnis vom Umlauf spanischer Handschriften in Mitteleuropa zu leisten, wobei die Versteigerungskataloge des Antiquars Leo

2. MORENO, Emilio: ,?La primera, hasta ahora desconocida, sonata para oboe y bajo continuo española? La sonata de Pla. Revista de musicología, 1986. S. 561. GERHARD, Robert: J. Pla Sonata III per a dos oboés, violins o flautes $i$ baix continu. Diputación de Barcelona - Biblioteca de Catalunya, Barcelona, 1996. Llorens, Josep María. S. 7.

3. SIEMENS HERNÁNDEZ, Lothar: Juan Oliver Astorga. Sonatas I-VI. Op. I para violín y bajo, siendo la $N^{\circ} 1$ también para flauta travesera, Londres, 1767. Sociedad Española de Musicología, Madrid, 1991. S. 4.

4. IRINA, Kriajeva: "Músicos españoles en Rusia: Vicente Martín y Soler en la Corte de Catalina II (en base a los materiales Rusos)". Anuario musical: Revista de musicología del C.S.I.C. Vol. XLIX. Barcelona, 1994. S. 191. GÁLVEZ, Genoveva: "Un nuevo hallazgo de música escénica de Vicente Martín y Soler: Il burbero di buon cuore". Revista de musicología, Vol. X/2. Madrid, 1987. S. 623. KLEINERTZ, Rainer: "Zwischen Neapel un Madrid: Vicente Martin y Soler und das spanische Königshaus”. Anuario musical: Revista de musicología del C.S.I.C., Vol. LI. Barcelona, 1996. S. 165. ROMERO NARANJO, Francisco Javier: "Martín y Soler. Una aportación a su producción religiosa". Revista de Musicología XXIII, 2. S. 135.

5. ROSENTHAL, Albi: „Liepmannssohn, Leo“. MGG, Band 8, Bärenreiter, 1960. S. 863. ROSENTHAL, Albi: „Die Lager kataloge des Musikantiquariats Leo Liepmannssohn (1866-1935). Festschrift Hans Schneider zum 60. Geburstag. Ed. R. Elvers und Ernst Vögel. München, 1981. S.193-218. HYATT KING, Alec: „Liepmannssohn, Leo“. New Grove, Vol. X, Macmillan 1980. S. 850. HYATT KING, Alec: „Liepmannssohn, Leo“. New Grove, Vol. XIV, Macmillan 2001. S. 686. 
Liepmannssohn die Hauptquelle sind. Auf diese Weise soll zur Erweiterung des Katalogs verschiedener spanischer Komponisten aus unterschiedlichen Epochen beigetragen werden. Gleichzeitig werden Hinweise auf das Vorkommen anderer - ebenfalls interessanter - Dokumente wie Briefe oder Abhandlungen gegeben.

Die Schwierigkeit bei den Nachforschungen über die Verbindung des Antiquariats Leo Liepmannssohns und den verkauften spanischen Handschriften ist, dass keine spezifischen Aufzeichnungen über dieses Thema existieren. Aus diesem Grund musste bei der ersten Annäherung an dieses Thema spezifiziert werden, wie viele spanische Handschriften und Abhandlungen Liepmannsohn insgesamt verkaufte und eine detaillierte Liste über deren Umlauf erstellt werden.

Um besagte Forschungen in einen Kontext zu bringen, wurden sekundäre Quellen verwendet.

Besondere Wichtigkeit kam dabei einer Nachforschung Sandra Myers ${ }^{6}$ über die Ankunft von deutschen „Einkäufern“ in Spanien, unter denen auch einer im Dienste Liepmannssohns stand, nach der „Desamortización“ zu. Zwar wird über die Entsendung derselben berichtet, die von ihnen getätigten Einkäufe werden jedoch nicht konkretisiert.

Ein anderer wichtiger Aspekt in Bezug auf das zu behandelnde Thema waren die zu dieser Zeit in Berlin stattfindenden spanischen Konzerte. Beim Studium der Konzertprogramme der „Singakademie und Philharmonie Berlin“ fiel auf, dass kein einziges Werk aus den von Liepmannssohn verkauften Handschriften aufgelistet war.

Nach Einsicht der Unterlagen Sandra Meyers und der Konzertprogramme verbleibt die Betrachtung des Nachlasses spanischer Musiker, die sich in dieser Periode in Deutschland aufhielten, mit dem Ziel, Hinweise auf besagte Handschriften zu finden. Unter all diesen Musikern kommt Enrique Fernández Arbós und Pablo de Sarasate besondere Bedeutung zu, die beide in großem Maße spanische Musik in Deutschland interpretierten. Keiner der beiden erwähnte jedoch die Verwendung des von Liepmannssohn verkauften Repertoires.

Das im Zentrum dieser Forschung stehende Antiquariat wurde in der zweiten Hälfte des 19. Jahrhunderts eröffnet, konkret in Paris im Jahre 1866, und schloss der Öffentlichkeit seine Türen in der ersten Hälfte des 20. Jahrhunderts. Das Antiquariat von Leo Liepmannssohn erreichte große Beliebtheit, weil es eine erhebliche Anzahl von Handschriften (vor allem Autographe) weltberühmter Komponisten wie Händel, J.S. Bach, Mozart und Beethoven versteigerte. Später griffen viele öffentliche Anstalten auf das besagte Antiquariat zurück, um einen Grossteil der zu versteigerten Handschriften in ihre Gewalt zu bringen. Unter anderem sind hier die Staatsbibliothek Berlin und das Archiv der Singakademie derselben Stadt zu nennen.

Leo Liepmannssohn eröffnete sein erstes Antiquariat um 1866 in Paris, zusammen mit seinem Geschäftspartner Dufour unter dem Namen „Liepmannssohn \& Dufour“ an der Rue des Saints-Péres

6. MYERS, Sandra: „Church or State? Implications of Liberal State Reform on 19th-Century Spanisch Music“. International Musicological Society. 17th International Congress. Programme Abstracts. Leuven, 1-7 August 2002. Abstract, S. 313. 
11. $\mathrm{Zu}$ einem späteren Zeitpunkt, während des Krieges, siedelten sie nach London um. Nach dem Krieg schließlich setzte der Antiquar seine Residenz definitiv in Berlin fest und verkaufte das Pariser Antiquariat um 1872 an die bekannte Buchhandlung J. Baur \& Détaille.

Im selben Jahr und wieder in der Hauptstadt Deutschlands schloss er sich mit den Antiquariern Asher \& Co. zusammen. Zwei Jahre später gründete er erneut sein eigenes Antiquariat. Um 1903 verkaufte er dieses jedoch an Otto Haas, der den Namen beibehielt, bis er um 1936 nach London emigrierte. In den letzten Jahren veröffentlichte Haas unter seinem eigenen Namen und mit dem Titel "Otto Haas Buchhandlung und Antiquriat", an der Motzstraße 75 in Berlin, eine ganze Reihe von Katalogen.

Das Antiquariat von Leo Liepmannssohn hatte während seiner Geschichte verschiedene Sitze. In der folgenden Tabelle sind sie chronologisch geordnet, da sie auch in den Katalogen zu finden sind.

\begin{tabular}{|c|c|c|}
\hline Jahr & Stadt & Adresse \\
\hline 1866 & Paris & 11 rue des Saints-Péres \\
\hline 1874 & Berlin & Markgraffengasse 52 \\
\hline 1883 & Berlin & Charlottenstr. 63 \\
\hline 1893 & Berlin & Berburgerstr. 14 \\
\hline
\end{tabular}

\section{Der Katalog des Antiquars Leo Liepmannssohn.}

Wie bereits erwähnt stützen sich diese Forschungen auf den sehr breiten von Leo Liepmannssohn veröffentlichten Katalog, der aus einer Sammlung von mehr als 300 Heften besteht. Sie sind vollständig in der Staatsbibliothek Preußischer Kulturbesitz zu Berlin unter der Signatur $A b$ 825. Liepmannssohn Antiquariats-Katalog einzusehen.

Die Kataloge führen, in alphabetischer Reihenfolge geordnet, alle Versteigerungen auf, die stattgefunden haben. Neben Handschriften und Musikabahndlungen sind darin auch Literatur und erstaunlicherweise sogar Portraits verschiedener Komponisten zu finden. Die Maße beinahe aller Kataloge sind 22,5 cm x 14,7 cm, mit Ausnahme des Katalogs 158, der 21,5 cm x $14 \mathrm{~cm}$ misst und des Katalogs 166, dessen Masse $22 \mathrm{~cm}$. x 14,5 cm sind.

Liepmannssohn veröffentlichte Jahr für Jahr eine unterschiedliche Anzahl von Katalogen, die von den stattgefundenen Versteigerungen und der Zahl versteigerter Werke abhing. Das ist der Grund, weshalb manche Jahre nahezu 8 Kataloge (wie beispielsweise um 1894) publiziert wurden, andere Jahre hingegen nur zwei oder drei (wie in 1877 und 1878).

Leo Liepmannssohn registrierte jedes einzelne Werk, indem er es nach Art (Handschrift oder Abhandlung) und Seitenzahl, nach Druckdatum (immer wenn es sich um ein schon vorher gedrucktes Werk handelte) und Versteigerungswert katalogisierte. Andere - beinahe wichtigere Daten - wie 
beispielsweise wem das Werk abgekauft und verkauft wurde, sucht man allerdings vergebens. Dies wäre höchst aufschlussreich gewesen, um den heutigen Verbleib jedes einzelnen Werks herauszufinden. Der Erhaltungszustand des im Berliner Archiv befindlichen Katalogs ist nicht sehr gut. Das lässt sich durch die für seine Herstellung benutzte Papierqualität und sein Alter erklären, weswegen auch viele Blätter teilweise zerstört und bei den einzelnen Heften die Blätter herausgefallen sind. Allerdings muss festgehalten werden, dass die Kataloge 158, 163, 166 und 170 eine bessere Papierqualität als der Rest aufweisen und deshalb besser erhalten sind.

Der Inhalt jedes einzelnen Katalogs ist nicht nur von herausragender Bedeutung für die spanische Musikwissenschaft sondern auch für die anderer Länder, wie die italienische, französische, portugiesische und deutsche, wegen des großen Versteigerungsangebots von Werken aus verschiedenen Ländern. Aus diesem Grund kann man sich sicher sein, dass sich hier außerordentlich wichtige Daten finden lassen, da man die Rezeption bestimmter Komponisten anhand des Kaufinteresses im 19. und zu Beginn des 20. Jahrhunderts untersuchen kann. Und nicht nur die Rezeption gewisser Komponisten kann dank der Kataloge untersucht werden. Auch die Möglichkeit einer Erweiterung der Werkkataloge einiger der versteigerten Komponisten ist gegeben.

\section{Die spanischen Handschriften}

Im Jahre 1900 veröffentlichte Liepmannssohn den Katalog 144 mit dem Titel „Spanische und Portugiesische Werke über Musik. Liturgie, Theater und Tanz“. Hiernach wurden in nur einem Jahr in Berlin die gewaltige Menge von 291 spanischen und portugiesischen Musikwerken versteigert ${ }^{7}$.

Weder bis zu jenem Zeitpunkt noch später war ein Handschriftenkatalog mit einem so konkreten Titel versehen worden, wie es dieser war. Nie publizierte Liepmannssohn einen Katalog, dessen Titel auf italienische, französische oder holländische Werke anspielte, sondern nur einen, der spanische und portugiesische Werke zum Inhalt hatte.

Die Herkunft vieler der Werke, die im Antiquariat versteigert wurden, kamen aus den Privatbibliotheken vieler zeitgenössischer Intellektueller, wie im Falle vom Nachlaß André, Alfred Bovet, F. Commer, R. Eitner, M. Friedländer, E. Goltermann, E. Grell, H. Kreutzschmar, V. Lasserré, R. Von Liliencron, James E. Matthew, E. Mörike, I. Möscheles, F. Mottl, W. Von Redern, H. Rieman, J. Rietz, F. W. Rust, E. F. Succi, Louis Spohr, M. Schlesinger, J. Stockhausen, W. Taubert, G. W. Teschener, E. Vogel, W. Wolffheim etc.

Im Katalog 144 wies Liepmannssohn auf die Herkunft vieler der versteigerten portugiesischen Handschriften hin, bei den spanischen tat er dies hingegen nicht. Im Katalog vermerkte er folgendes:

7. ROMERO NARANJO, Francisco Javier: „La circulación de manuscritos portugueses en el anticuario de Leo Liepmannssohn en París y Berlín“. Nassarre. Revista Aragonesa de Musicología, XVII, 1-2. Institución „Fernando el Católico“, 2001. S. 339.

Anuario Musical, 59 (2004) 
"La plupart des articles portés sur ce catalogue proviennet de la bibliothéque du savant musicologue Portugais Mr. Joaquin de Vasconcellos auteur de l'ouvrage "Os Musicos Portuguezes. Biographia - Bibliographia" (Porto, 1870, 2 volumes), et d'autres ouvrages d'erudition musicale.

$\mathrm{Mr}$ de Vasconcellos a publié lui-même le catalogue raisonné de sa bibliothéque (Catalogue des livres rares composant la bibliothéque musicale d'un amateur. Porto, 1898, gr. In 8, de 193 pages) et les descriptions ou notices en langue portugaise que j’ai jointes à un certain nombre d'articles sont copiées de ce même catalogue".

Im Verzeichnis spanischer Werke, die in dieser Forschungsarbeit vorgestellt werden, wird nicht nur bekannt gegeben, was im Katalog 144, sondern auch, was im Gesamtkatalog versteigert wurde, da häufig und regelmäßig eine große Anzahl spanischer Werke dabei war. Das heißt, dass Liepmannssohn nicht abwartete bis er eine größere Anzahl spanischer und portugiesischer Werke in seinem Besitz hatte, um einen speziellen Katalog zu schaffen. Vielmehr scheint er diese fortwährend und unmittelbar nach dem Einkauf versteigert zu haben. Daher finden sich in den Jahren 1904, 1911, 1913, 1923 und 1930 über 170 versteigerte Werke spanischer Komponisten, die fast ausschließlich in Spanien gearbeitet haben, wie beispielsweise Gaetano Bruneti.

Es wurde bereits erwähnt, dass Liepmannssohn nicht angab, wem er die Handschriften abkaufte und wem er sie wieder verkaufte. Ein Stammkunde war das Berliner Archiv. Deshalb kann festgestellt werden, dass die Daten der Versteigerung mit derjenigen der Ankunft im Berliner Archiv übereinstimmen, so am Beispiel der Abhandlungen von Bermudo, von Salinas, den Briefen von Sarasate oder den Werken von Gaetano Brunetti, die im besagten Archiv gesammelt wurden. Als Folge des 2. Weltkriegs verschwand ein Großteil davon, wodurch sich ihre Spur verliert. Sie wurden vermutlich gestohlen oder verbrannt.

Das in diesem Artikel vorgestellte Werkeverzeichnis wurde folgendermaßen strukturiert:

Als Erstes sind die Briefe in alphabetischer Reihenfolge aufgelistet und nicht nach entsprechender Epoche klassifiziert, da es nur deren 10 sind, folglich eine ziemlich geringe Zahl. Anschließend und bevor die Handschriften und Abhandlungen aufgelistet werden, kann das David Pérez zugeschriebene Portrait betrachtet werden. Dieser Musiker war zwar kein Spanier sondern nur spanischer Abstammung wie Carlos Ordóñez und Marianna Martines, aber da er sich im Katalog über spanische Musiker befindet, wurde er hier ebenfalls miteingeschlossen.

Die versteigerten Werke sind nach Jahrhunderten unterteilt worden, wobei, um Verwirrungen zu verhindern, zusätzlich einerseits nach Abhandlungen, anderseits nach Handschriften strukturiert wurde. Erstaunlich erscheint, dass weit mehr Abhandlungen versteigert wurden als Handschriften, insbesondere im 16. und 17. Jahrhundert. Dennoch lassen sich auch hier Ausnahmen finden, wie im Falle der Versteigerung einer großen Anzahl von Gaetano Brunetti-Werken im Vergleich mit denen anderer Komponisten.

Unter den wichtigsten Aspekten befinden sich die versteigerten Briefe verschiedener Komponisten, so im Falle von Pablo de Sarasate, dessen Korrespondenz zu großen Teilen versteigert wurde und die sich in der Gegenwart im Berliner Archiv befindet. In den Briefen wird viel von den Konzerten de Sarasates in Frankreich und Deutschland gesprochen. Sie enthalten auch interessante dokumentarische Information. 
Der Umlauf der spanischen Handschriften aus dem Antiquariat Leo Liepmannssohns in Paris und Berlin

Die durch Liepmannssohn versteigerte Korrespondenz, die sich jedoch nicht im Berliner Archiv befindet, ist dennoch sehr wichtig, wie die Briefe von Arteaga, Brunetti, Barbieri, Pau Casals und sogar Dionisio Aguado beweisen.

Vom Gitarristen und Komponist Dionisio Aguado wurde ein Brief versteigert, von dem man bisher keine Kenntnis hatte; diese Korrespondenz befindet sich nicht im Berliner Archiv. Dennoch scheint der Inhalt wichtig gewesen zu sein, zumal sie für 10 Mark verkauft wurde; ein relativ hoher Preis für einen Brief der erst noch aus nur einer Seite bestand.

Die Handschriften und Abhandlungen des 16. Jahrhunderts wurden im Laufe der letzten Jahre des 19. Jahrhunderts und anfangs des 20. Jahrhundert versteigert. Unter ihnen waren die Abhandlungen die meist verkauften und auch die, wofür am meisten geboten wurde. Insgesamt wurden 10 Musikabhandlungen der repräsentativsten zeitgenösischen Autoren wie Francisco Salinas, Juan Bermudo, Tomás Santa María und Melchor de Torres versteigert. Dabei ist die Versteigerung des Libro de música práctica von Francisco Tovar hervorzuheben, für dessen Erwerb 800 Mark bezahlt wurden.

Heutzutage gibt es keine Dokumentation über die Abhandlungen Juan Martínez de Rigo und Pedro Ruyz Alcoholado, die beide mit dem liturgischen Zeremoniell und Gregorianischem Gesang in Verbindung gebracht werden.

Was die musikalischen Kompositionen betrifft, wovon lediglich zwei versteigert wurden, darf das Werk von Luis de Narváez nicht vergessen werden. Es wurden 4800 Mark (ein ganzes Vermögen) für Los seis libros del Delphin de música de cifras para tañer la vihuela bezahlt. Das ist der höchste Preis, der jemals in den Liepmannsohnschen Versteigerungen für eine spanische Handschrift bezahlt wurde. Bei diesem Werk von Narváez fällt auf, dass laut Katalog 78 nur der erste Band für 1000 Mark verkauft wurde und erst später die sechs Werke (Gesamtausgabe) für die genannten 4800 Mark. Der Käufer war einmal mehr das Berliner Archiv, da in dieser Institution die Herkunft des Erwerbs und der heutige Standort zu finden sind.

Die Versteigerung der Handschriften und Abhandlungen aus dem 17. Jahrhundert konzentrierten sich fast vollständig auf die Abhandlungen jenes Jahrhunderts, mit der Ausnahme der Versteigerung der Werke von Correa de Arauxo im Jahr 1869, wie es im Katalog 15 nachzulesen ist. Auch für sie wurde eine ziemlich hohe Summe bezahlt, 400 Mark, in den Anfängen des Antiquariats, als es noch in Paris stand.

Über die Abhandlungen des 17. Jahrhunderts gibt es eine, über die keinerlei Untersuchungen gefunden wurden. Es handelt sich um eine Abhandlung zu Gitarre und vandola, von der man bisher keine Kenntnis hatte und deren Autor Joseph Bro ist. Das Werk trägt den Titel Guitarra española y vandola en dos maneras de guitarra, castellana y catalana de cinco ordenes, la qual se enseña a temblar, y tañer rasgado, todos los puntos naturales, y mollados, con estilo maravilloso und wurde 1639 in Gerona veröffentlicht. Der aktuelle Standort dieser Abhandlung ist unbekannt, da sie vermutlich von einer Privatperson erworben wurde. Der Versteigerungskatalog von Liepmannssohn bekräftigt jedoch seine Existenz ${ }^{8}$. Der Preis, für den sie 1893 versteigert wurde, war mit 180 Mark

8. Befindet sich nicht in der Staatsbibliothek Preußischer Kulturbesitz zu Berlin 
nicht sehr hoch, was aber nicht bedeutet, dass sie weniger wert war, sondern wahrscheinlich eine gewisse Unkenntnis seitens des Antiquars bestand.

Die Versteigerungen von Handschriften und Abhandlungen aus dem 18. Jahrhundert zeigen eine größere Anzahl vom Kompositionen als von zeitgenössischen Abhandlungen. Bevor die bedeutendsten Werke, die versteigert wurden, kommentiert werden, soll darauf aufmerksam gemacht sein, dass eine Großzahl von Kompositionen nichtspanischer Autoren, die aber lange Zeit in Spanischen Landen gelebt haben, miteingeschlossen sind. Darunter Gaetano Brunetti mit mehr als 150 versteigerten Werken, Mauro D’Alay mit 13 versteigerten Werken und David Pérez mit sechs.

Eine der versteigerten Abhandlungen erregt speziell Aufmerksamkeit, weil sie über Crotalogie spricht oder die Wissenschaft der Kunst Kastagnetten zu spielen. Sie wurde 1792 in Madrid veröffentlicht, unter dem Titel Crotalogía o ciencia de las castañuelas. Instrucción científica del modo de tocar las castañuelas para baylar el bolero y poder facilmente y sin necesidad de maestro acompañarse en todas las mudanzas. Das Werk wurde um 1900 laut Katalog 144 für 15 Mark versteigert ${ }^{9}$.

Ein weiteres Buch, das zu einem ziemlich hohen Preis versteigert wurde war das von Roel del Río zum Preis von 450 Mark um 1930. Dieses Buch scheint Eindruck gemacht zu haben, da in demselben Katalog die folgende Bewertung gemacht wurde:

"Sehr interessantes spanisches Lehrbuch der Musik. Nach 18 Bl. Dedikationen, Privilegien etc. Beginnt das Werk mit der "Dissertation preliminar..."

Der Großteil der Versteigerungen der Dokumente aus dem 19. Jahrhundert fanden um 1900 statt und wurden aus diesem Grund im Katalog Nummer 144 aufgeführt. In ihm werden die Abhandlungen und didaktischen Methoden des Solfeggierens von Instrumenten gesammelt, wo vor allem die neuesten in Spanien getätigten Publikationen von Hilarión Eslava, Francisco Asenjo Barbieri, José Inzenga, Felipe Pedrell und Antonio Peña y Goñi erschienen. Dies alles führt zur Folgerung, dass man in Deutschland über alles, was in Spanien an Musikmaterial veröffentlicht wurde auf dem Laufenden war und dass ein viel größeres Interesse daran bestand als ursprünglich angenommen.

Zusammenfassend kann statuiert werden, dass besagte Handschriften durch die Arbeit der „Einkäufer“ nach der Periode der spanischen „Desamortización“ nach Deutschland gelangten. Einerseits wurden Handschriften in Berlin durch Leo Liepmannssohn verkauft, andererseits sind dort nur sehr wenige derselben aufzufinden.

Hervorzuheben ist der scheinbar hohe Wert, der diesen Handschriften um 1900 in Deutschland zukam.

9. Es ist bekannt, dass der Autor dieses Werkes unter dem Pseudonym Francisco Agustín Florencio schrieb, obschon sein richtiger Name Juan Fernández de Rojas war. Das Werk ist an sich nicht ein Lehrwerk über das Spielen der Kastagnetten, sondern ein Spott über die Regeln des Neuklassischen Theaters, d.h. ein Verspotten des Französierenden, das in der zeitgenössischen spanischen Gesellschaft vorherrschte. Es war eine Satire der wissenschaftlichen Methoden der französischen Enzyklopädisten, der rigiden Tendenzen der klassischen Schule, der Regeln der drei Einheiten des Theaters etc. Das Buch wurde vielfach aufgelegt und seit seiner Ersterscheinung von zahlreichen Polemiken umgeben. Wie man sieht, interessierte man sich auch in Deutschland für das Werk. 
Die Untersuchung von Handschriften und Abhandlungen, die im Antiquariat verkauft wurden regt ernsthaft zum Nachdenken über die große Rezeption und das Interesse an der spanischen Musik in Deutschland an. Dieses ist nicht nur auf die Käufe öffentlicher Anstalten (die recht groß war) zurückzuführen, sondern auch auf Privatkäufer, die sich den Luxus leisten konnten, große Geldsummen für den Kauf einer spanischen Handschrift oder Abhandlung auszugeben.

Es folgt nun die vollständige Auflistung der im Antiquariat Leo Liepmannssohns versteigerten spanischen Werke:

\section{Briefe}

\section{Arteaga, Esteban de}

Brief vom 24 Juni 1783.

Katalog 190.

Jahr 1916.

Enthält zwei Seiten

In Berlin für 15 Mark versteigert

\section{Aguado, Dionisio}

Brief auf Französisch.

Katalog 174.

Jahr 1910.

Enthält eine Seite

In Berlin für 10 Mark versteigert

\section{Barbieri, Francisco Asenjo}

Brief auf Französisch.

Katalog 141.

Jahr 1869.

Enthalt zwei seiten.

In Berlin für 4 Mark versteigert.

\section{Brunetti, Gaetano}

Lettre autographe signeé.

Katalog 99.

Jahr 1893.

In Berlin für 1 Mark versteigert.

\section{Casals, Pablo}

Carta escrita el 15 de noviembre.

Katalog 61.

Jahr 1888.

In Berlin für 7 Mark versteigert.
Sarasate, Pablo de

Carta en francés a Goldschmidt.

Katalog 48.

Jahr 1886.

In Berlin für 1 Mark versteigert.

Carta en francés a Goldschmidt.

Katalog 48.

Jahr 1886.

In Berlin für 3 Mark versteigert.

Carta en italiano escrita en Viena a un "Caro

Maestro".

Katalog 48.

Jahr 1886.

In Berlin für 1 Mark versteigert.

Correspondencia a Otto Goldschmidt.

Katalog 174.

Jahr 1910.

In Berlin für 15 Mark versteigert.

Correspondencia.

Katalog 215.

Jahr 1909

Enthält eine seite.

In Berlin für 10 Mark versteigert

\section{Portraits}

Perez, David

Portrait - Medaillon in reich ornamentierte Einfassung.

Katalog 86.

Jahr 1890. 
In Berlin für 8 Mark versteigert.

\section{Sarasate, Pablo de}

Portrait. Visitphotographie. Francfurt, 17 Déc. 1878.

Katalog 215

Jahr

In Berlin für 12 Mark versteigert.

\section{Jh.}

\section{Abhandlungen}

Alcoholado, Pedro Ruyz

Ceremonial romano para misas cantadas y rezadas...

Alcalá, 1589.

Katalog 144.

Jahr 1900

In Berlin für 15 Mark versteigert.

\section{Bermudo, Fray Juan}

Comienza el libro llamado declaración de instrumentos musicales... Ossuna, 1555.

Katalog 137.

Jahr 1899.

In Berlin für 400 Mark versteigert

Comienza el arte Tripharia dirigida a la yllustre... Ossuna, 1555.

Katalog, 137.

Jahr 1899.

In Berlin für 500 Mark versteigert.

\section{Durán, Domingo}

Lux bella. Ars cantus plani composita breuissimo compendio Lux bella nuncupata per baccalarium dominicum duranium: \& clarissimo dno petro ximenio cauriensi episcopo reurendisimo: atq; sacratissime theologie peritissimo dedicata feliciter incipitur ad laudem dei. Sevilla, 1492.

Katalog 144.

Jahr 1900

In Berlin für 600 Mark versteigert

\section{Martínez de Rigo, Juan}

Arte de canto llano y reduzida nuevamente a su entera perfeccion: según la practica del canto llano. Alcalá de Henares, 1532.
Katalog 52.

Jahr 1899.

In Berlin für 180 Mark versteigert

\section{Montanos, Francisco}

Arte de música, theorica y practica de Francisco Montanos. Valladolid, MDXCII

Katalog 137.

Jahr 1899.

In Berlin für 180 Mark versteigert.

Arte de canto llano con entonaciones de coro y altary otras cosas. Nuevamente corregido y aumentado. El arte practico de canto, de órgano, con motetes, o lecciones diversas, por todos los tiempos, y claves, por Don José de Torres. Madrid, 1712.

Katalog 23.

Jahr 1870.

In Frankreich für 80 Fränkisch versteigert

\section{Morales, Cristobal}

Magnificat cum qvatvor vocibus. Venetiis, apud Franciscum Rampazetum, 1563.

Katalog 221.

Jahr 1930.

Enthält 30 seiten.

In Berlin für 60 Mark versteigert.

\section{Narvaez, Luis de}

El primer libro del Delphin de música de cifras para tañer la vihuela.

Katalog 78.

Jahr 1889.

In Berlin für 1000 Mark versteigert

Los seis libros del Delphin de música de cifras para tañer la vihuela.

Katalog 183.

Jahr 1913.

In Berlin für 4800 Mark versteigert.

\section{Ortiz, Diego de}

Tratado de glosas sobre clausulas y otros géneros de puntos en la música de violines. Roma, 1518.

Katalog 218.

Jahr 1927.

In Berlin für 200 Mark versteigert. 
Tratado de glosas sobre clausulas y otros géneros de puntos en la música de violines. Roma, 1518. Herausgeg. V. Max Scheneider.

Katalog 214

In Berlin für 4 Mark versteigert.

\section{Salinas, Francisco}

De musica libri septem. Salmanticae, 1577.

Katalog 71.

Jahr 1888.

In Berlin für 150 Mark versteigert.

De musica libri septem. Salmanticae, 1577.

Katalog 221.

Jahr 1930.

In Berlin für 500 Mark versteigert.

\section{Tapias, Martin}

Vergel de música espiritual especulativa y activa, del qual muchas diversas y suaves flores se pueden coger. Burgos de Osma, 1570

Katalog 156.

Jahr 1905.

In Berlin für 200 Mark versteigert

\section{Santa María, Tomás de}

Libro llamado arte de tañer fantasia asi para tecla como para vihuela, y todo instrumento, en que se pudiere tañer a tres, y quatro vozes, y a mas. Valladolid, 1565.

Katalog 167.

Jahr 1908.

In Berlin für 700 Mark versteigert.

\section{Torres, Melchor de}

Arte ingeniosa de música con nueva manera de auizos breues y compendios sobre toda la facultad de ella. Alcalá, 1544.

Katalog 156.

Jahr 1905.

In Berlin für 40 Mark versteigert.

\section{Tovar, Francisco}

Libro de música práctica. Compuesto por mosz. Francisco Tovar: dirigido al ilustrisimo y reverendisimo sehnor don Enrique de Gardoa obispo de Barcelona... Barcelona, 1510.

Katalog 137.
Jahr 1899.

In Berlin für 800 Mark versteigert.

\section{Jh.}

\section{Abhandlungen}

\section{Bro, Joseph}

Guitarra española y vandola en dos maneras de guitarra, castellana y catalana de cinco ordenes, la qual se enseña a temblar, y tañer rasgado, todos los punto naturales, y mollados, con estilo maravilloso... Gerona, 1639.

Katalog 103.

Jahr 1893.

In Berlin für 180 Mark versteigert.

\section{Cerone, Pedro}

El Melopeo y maestro tratado de música theorica y practica.

Katalog 144.

Jahr 1900

In Berlin für 900 Mark versteigert.

\section{Correa de Arauxo, Francisco}

Libro de tientos y discursos de música práctica, y theorica de organo, intitulado facultad orgánica. Alcalá, 1626.

Katalog 15.

Jahr 1869.

In Frankreich für 400 Fränkisch versteigert.

Libro de tientos y discursos de música práctica, y theorica de organo, intitulado facultad orgánica. Alcalá, 1626.

Katalog 154.

Jahr 1904

In Berlin für 850 Mark versteigert.

\section{Esquivel Navarro, Juan}

Discurso sobre el arte del dançado y sus excelencias $y$ primer origen, reprobando las acciones deshonestas. Sevilla, 1642.

Katalog 23.

Jahr 1870.

In Frankreich für 100 Fränkisch versteigert 


\section{Lorente, Andrés}

El porqué de la música, en que se contiene los quatro artes de ella, canto llano, canto de órgano, contrapunto, y composición. Alcalá de Henares, 1672.

Katalog 175.

Jahr 1910.

Enthält 695 seiten.

In Berlin für 160 Mark versteigert.

El porqué de la música, en que se contiene los quatro artes de ella, canto llano, canto de órgano, contrapunto, y composición. Alcalá de Henares, 1672.

Katalog 221.

Jahr 1930.

Enthalt 695 seiten.

In Berlin für 180 Mark versteigert.

\section{Monserrate, Andrés}

Arte breve y compendiosa de las dificultades que se ofrecen en la música practica del canto llano. Valencia, 1614.

Katalog 144.

Jahr 1900.

In Berlin für 20 Mark versteigert

\section{Ruiz de Ribayaz, Lucas}

Luz y norte musical para encaminar por las cifras de la guitarra española, y arpa, tañer y cantar a compás de órgano. Madrid 1677.

Katalog 90.

Jahr 1891.

In Berlin für 140 Mark versteigert.

\section{Sanz, Gaspar}

Instrucción de música sobre guitarra Española, y método de sus primeros rudimentos, hasta tañerla con destreza. Zaragoza, 1697.

Katalog 183.

Jahr 1913.

In Berlin für 265 Mark versteigert.

Instrucción de música sobre guitarra Española, y método de sus primeros rudimentos, hasta tañerla con destreza. Zaragoza, 1697.

Katalog 114.

Jahr 1895.

In Berlin für 250 Mark versteigert.

\section{Jh.}

\section{Werke}

\author{
Alay, Mauro de \\ Cantate a voce sola e Suonate a violino solo col \\ Basso, dedicate a Carlos Lenos, Duca di \\ Richmond.. \\ Katalog 154. \\ Jahr 1904. \\ Enthält 2 seiten. \\ In Berlin für 17 Mark versteigert.
}

XII Concerti a violino principale, violino primo $e$ secondo, alto, viola, violoncello e cimbalo. Opera Omnia amsterdam. (6 Separate parts, complete)

Katalog 154.

Jahr 1904.

In Berlin für 27 Mark versteigert.

\section{Oliver y Astorga, Juan}

Stabat Mater a 4 voci con stromenti.

Katalog 82.

Jahr 1890.

53 seiten.

In Berlin für 3 Mark versteigert.

Stabat Mater für 4 Singstimmen.

Katalog 82.

Jahr 1890.

In Berlin für 2,50 Mark versteigert.

\section{Brunetti, Gaetano}

Sinfonia von Violini, Oboes, Corni, Viola, Fagotto e Basso. $N^{\circ} 23$. Dat. 1783.

Katalog 228.

Jahr 1923.

Enthält 44 seiten.

In Berlin für 60 Mark versteigert.

Sinfonia con Violini, Oboes, viola, fagotto, cornie Basso. $N^{\circ} 16$. Originale.

Katalog 215.

Jahr 1923.

Enthält 47 seiten.

In Berlin für 40 Mark versteigert. 
Sinfonia in C Minore con violini, Oboe, Corni, Viola, Faggotto obligatto e Basso.

Katalog 215.

Jahr 1923.

Enthält 52 seiten.

In Berlin für 40 Mark versteigert.

Aria: "Quanti amor ferisce". Libro de arias, 16 arias.

Katalog 216.

Jahr 1923.

Enthält 214 seiten.

In Berlin für 24 Mark versteigert.

Divertimento Quarto a due violini. Tutto per vso del

Exmo S. Dca d'Alba.

Katalog 174.

Jahr 1910.

Enthält 6 seiten.

In Berlin für 65 Mark versteigert.

Variazioni pour orchestre.

Katalog 174.

Jahr 1910.

Enthält 15 seiten.

In Berlin für 85 Mark versteigert.

Sinfonie für 2 Violinen, 2 oboen, 2 corni, 2 fagotti, viola, Basso. $N^{\circ} 27$. Dat. 1787.

Katalog 237.

Jahr 1934.

Enthalt 44 seiten.

In Berlin für 60 Mark versteigert.

Seis overturas.

Katalog 237.

Enthält 190 seiten.

Jahr 1934

In Berlin für 60 Mark versteigert.

6 TRIO per 2 Violini e Violoncello dedicatti a Don Carlo Principe d'Asturias. Opera III, libro secondo di trio.

Katalog 169.

Jahr 1907

In Berlin für 11 Mark versteigert.

Divertissemens pour violon, alto et violoncelle. Katalog 154.
Jahr 1904.

In Berlin für 43 Mark versteigert.

Collection 50 Quatuors pour 2 violons, alto et Violoncelle. 4 vols.

Katalog 154.

Jahr 1904.

In Berlin für 217 Mark versteigert.

Collection de 43 Quintetti, dont 6 pour 2 violons, alto et 2 violoncelles, 6 pour 2violons, alto, basson et Violoncelle. 5 vols.

Katalog 154.

Jahr 1904.

In Berlin für 268 Mark versteigert.

Sei SESTETTI per tre violini, viola e due violoncelli obligatti. Opera I.

Katalog 154.

Jahr 1904.

In Berlin für 4 Mark versteigert.

Sinfonia con violini, oboe, corni, viola, fagotto $e$ basso. $N^{\circ} 12$.

Katalog 48.

Jahr 1926.

Enthält 64 seiten.

Divertimento secondo a Due Violini. Fatto per uso del exmo. S. Duca d'Alba

Composto da Brunetti.

Katalog 41.

Jahr 1913.

Il Maniatico. Sinfonia a violini, Oboes, Corni, Viola, Fagotto, Violoncello obligato e Basso. Originale 1780.

Katalog 46.

Jahr 1921.

Enthäalt 51 seiten.

Duetto (B-dur) a Due Violini fatto per l'Illustrisimo

Sig. Dr. Francesco Ant. Pavisien de Gaetano Brunetti 766". Dat. 1766.

Katalog 47.

Jahr 1921.

Enthält 4 seiten. 
Sammlung 18 Divertimente. 6 per violino, viola, $e$ violoncello; 6 per camara; 6 per Violino, viola, Basso.

Katalog 47.

Jahr 1921.

Enthält 111 seiten.

Quartetto I-IV a due violini, viola, e Basso fatti per l'exmo S. Ducha D’Alba.

Katalog 47.

Jahr 1921.

Enthält 67 seiten.

Sinfonia in Bemoll. Con Violini, oboe, corni, trombbe, viola, Fagotto e Basso. $N^{\circ} 19$.

Katalog 50.

Jahr 1927.

Enthält 48 seiten.

Sinfonia (Es dur) con Violini oboe corni, viola, Fagotto e Basso. Originale $N^{\circ} 21$.

Katalog 52.

Jahr 1928.

Enthält 62 seiten.

Sinfonia con violini, Oboe, corni, fagotto, viola, $e$ Basso. $N^{\circ} 24$. Dat. 1783.

Katalog 61.

Jahr 1930.

Enthält 59 seiten.

Marcha Maestoso. Para las parejas de aranjuez del año 1787.

Katalog 47.

Jahr 1907.

Enthält 17 seiten.

Marcha Primavera para las parejas de aranjuez del año 1788.

Katalog 48.

Jahr 1909.

Enthält 22 seiten.

\section{Martín y Soler, Vicente}

L'arbore de Diana.

Katalog 58.

Jahr 1887.

In Berlin für 1,50 Mark versteigert.
VI Canoni a tre voci col accomp. di cembalo

Katalog 58.

Jahr 1887

Enthält 23 seiten.

In Berlin für 2 Mark versteigert.

L'arbore de Diana.

Katalog 221.

Jahr 1930.

In Berlin für 100 Mark versteigert.

Il Burbero di buon Cuore. Dramma Giocoso (in 2 atti). Rappresentato nel Teatro del Corte a Vienna l'Anno 1787. Si vende in Venezia da domenica Melliga, copista del Teatro di S. Moisé.

Katalog 221.

Jahr 1930.

2 Bande.

In Berlin für 100 Mark versteigert.

\section{Martines, Marianna}

Briefe

Katalog 215

Jahr

In Berlin für 24 Mark versteigert.

E.B.m.U. vienna, 16 febr. 1784. 2 S. $4^{\circ}$ (Italienisch) Mit adresse . Von größter Seltenheit "Sehr schöner Brief an Padre G. B. Martini, dem sie ihr Portrait übersendet"

Briefe

Katalog 228.

\section{Pérez, David}

Cantata a 4 voci con stromenti, 1749. 2 vol.

Katalog 105.

Jahr 1894.

In Berlin für 25 Mark versteigert.

Mattutino di morti, 1774.

Katalog 105.

Jahr 1894.

In Berlin für 6 Mark versteigert.

Solfeggi a due voci col Basso.

Katalog 134.

Jahr 1898

Enthält 160 seiten. 
In Berlin für 6 Mark versteigert.

\section{Abhandlungen}

\section{Aragón, Frutos Bartholomé}

Ceremonial Romano de la Misa rezada conforme el Missal mas moderno. Madrid 1707.

Katalog 144.

Jahr 1707.

In Berlin für 4 Mark versteigert.

\section{Bails, Benito}

Lecciones de clave, y principios de harmonia. Madrid, 1775.

Katalog 144.

Jahr 1900.

In Berlin für 3 Mark versteigert.

\section{Bustamante, Juan de}

Tratado de ceremonias de la misa. Barcelona, 1705.

Katalog 144.

Jahr 1900.

In Berlin für 3 Mark versteigert.

\section{Comes y de Puig, Berardo}

Fragmentos músicos caudalosa fuente gregoriana, en el arte de canto llano. Barcelona, 1739.

Katalog 175.

Jahr 1910.

Enthält 197 seiten

In Berlin für 50 Mark versteigert.

\section{Eximeno, D. Lazarillo Vizcardi}

Sus investigaciones músicas con ocasión del concurso a un magisterio de capilla vacante. Dalas a la luz la sociedad di bibliofilos españoles. Madrid, 1872-73. Katalog 103.

Jahr 1893.

In Berlin für 50 Mark versteigert.

Del origen y reglas de la músicacon la historia de sus progresos, decadencia y restauración. Obra escrita en italiano por el abate Don Antonio Eximeno, y traducida al castellano por $D$. Francisco Antonio Gutierrez. Madrid, 1796. 3 vol. Katalog 107.

Jahr 1894

In Berlin für 12 Mark versteigert.

\section{Florencio, Francisco Agustín}

Crotalogía o ciencia de las castañuelas. Instrucción científica del modo de tocar las castañuelas para baylar el bolero y poder facilmente y sin necesidad de maestro, acompañarse en todas las mudanza de que est ja adornado este gracioso bayle español. Parte primera contiene una noción exacta del instrumento llamado castañuelas, su origen, modo de usarlas, y los preceptos elementales reducidos a riguroso método geométrico, juntamente con la invención de las castañuelas armónicas, que se pueden templar y arreglar con los demás instrumentos. Madrid, 1792.

Katalog 144.

Jahr 1900.

In Berlin für 15 Mark versteigert.

\section{Herrando, José}

Método de violín. Madrid, 1756.

Katalog 23.

Jahr 1870.

In Frankreich für 24 Fränkisch versteigert.

\section{Martín y Coll, Antonio}

Arte de canto llano y breve resumen de sus principales reglas para cantores de coro, dividido en 2 libros; en el primero se declara que pertence a la Theorica; y en el segundo lo que se necesita para la práctica y las entonaciones dde los Psalmos con el Organo. Madrid, 1714.

Katalog 144.

Jahr 1900.

In Berlin für 30 Mark versteigert.

\section{Nasarre, Pablo}

Fragmentos músicos repartidos en quatro tratados, en que se hallan reglas generales y muy necesarias... Madrid, 1700.

Katalog 85.

Jahr 1890.

In Berlin für 55 Mark versteigert.

Escuela de música, según la practica moderna. Zaragoza, 1724.

Katalog 100.

Jahr 1893.

In Berlin für 150 Mark versteigert. 
Fragmentos músicos repartidos en quatro tratados, en que se hallan reglas generales y muy necesarias... Madrid, 1700.

Katalog 221.

Jahr 1930.

In Berlin für 60 Mark versteigert.

\section{Roel del Río, Antonio Ventura}

Institución armónica, ó doctrina musical, theorica, y practica, que trata del canto llano, y de Organo. Madrid, por los hered. De la Viuda de Juan García Infanzón, 1748.

Katalog 221.

Jahr 1930.

In Berlin für 450 Mark versteigert.

\section{Romero de Avila, Gerónimo}

Arte de canto llano y organo ó promptuario musico, div. en quatro partes. La primera trata de la especulativa del canto llano. La segunda de la practica del mismo canto. La tercera, de la especulativa, y practica de todo hymno, secuencia o prosa. Y laquarta, o de la especulativa y practica del canto de órgano, según el moderno estilo. Madrid, 1785.

Katalog 23.

Jahr 1870.

In Berlin für 50 Mark versteigert.

\section{Soler, Antonio}

Llave de la modulación y antiguedades de la música: teorica y practica para el mas claro conocimiento de cualquier especie de figuras, desde el tiempo de Juan de Muris hasta hoy, con algunos canones enigmáticos, y sus resoluciones.

Katalog 23.

In Frankeich für 45 Fränkisch versteigert.

\section{Traveria, D.}

Ensayo gregoriano o estudio practico del canto llano y figurado ilustrado con algunas cosas curiosas para el aprovechamiento y enseñanza de los que siguen los concursos en las santas iglesias de España, 1794.

Katalog 37 .

Jahr 1885.

\section{Torres Martínez Bravo, José de}

Reglas generales de acompañar, en órgano, clavicordio, y harpa, con solo saber cantar la parte, $o$ un baxo figurado. Madrid, 1702.

Katalog 197

Jahr 1917.

Enthält 143 seiten

In Berlin für 180 Mark versteigert.

Reglas generales de acompañar, en órgano, clavicordio, y harpa, con solo saber cantar la parte, o un baxo figurado. Madrid, 1702.

Katalog 221.

Jahr 1930.

Enthält 124 seiten

In Berlin für 115 Mark versteigert.

Yriarte, Tomás de

La música, poema. Madrid, 1779.

Katalog 23.

Jahr 1870.

In Frankreich für 10 Fränkisch versteigert.

\section{Jh.}

\section{Abhandlungen}

\section{Aguirre, B.}

Juego de versos para visperas sobre los 8 tonos del canto llano, para órgano. Madrid.

Katalog 144.

Jahr 1900.

In Berlin für 2,50 Mark versteigert.

\section{Albéniz, Pedro}

Método completo de piano del conservatorio de músi-

ca.

Enthält 200 seiten.

Katalog 144.

Jahr 1900.

In Berlin für 6 Mark versteigert.

\section{Andrevi, Francisco}

Tratado teórico práctico de armonía, contrapunto, fuga, melodía, instrumentación, composición. Madrid 1848. 
Katalog 144.

Jahr 1900.

In Berlin für 5 Mark versteigert.

\section{Aranguren, José}

Prontuaruio del cantante e instrumentista. Madrid 1860.

Katalog 144.

Jahr 1900.

In Berlin für 2,50 Mark versteigert.

\section{Baños, Melitón}

Ofertorio-fuga orgánica para órgano. Madrid.

Katalog 144.

Jahr 1900.

In Berlin für 2 Mark versteigert.

\section{Barbieri, Francisco Asenjo}

Cancionero musical de los siglos XV y XVI. Madrid Enthält 636 seiten.

Katalog 85.

Jahr 1890.

In Berlin für 30 Mark versteigert.

Las castañuelas. Estudio jocoso dedicado a todos los boleros y danzantes por uno de tantos. Madrid 1879.

Katalog 140.

Jahr 1899.

In Berlin für 15 Mark versteigert.

Discurso leído en la academia de bellas artes de San Fernando en la sesión del 10 de mayo de 1874 para solemnizar la agregación de la sección de música. Madrid 1874.

Katalog 9.

Jahr 1876.

In Berlin für 3 Mark versteigert

\section{Barreto, José Parada}

Guía musical e instrucciones sobre los requisitos y cualidades necesarias para emprender y seguir con éxito las diferentes carreras de la música. Madrid 1866.

Katalog 144.

Jahr 1900.

In Berlin für 10 Mark versteigert.

\section{Benito, C.}

Siete palabras para tiple, tenor, bajo, y coro con acompañamiento de órgano expresivo. Madrid 1872.

Katalog 144.

Jahr 1900

In Berlin für 4,50 Mark versteigert.

\section{Benito, José de Cosme}

Método elemental de Violoncello. Op. 133. Madrid.

Katalog 144.

Jahr 1900.

In Berlin für 2,50 Mark versteigert.

\section{Calvo, Julián}

Seis pequeños motivos del himno Pange Lingua para órgano. Madrid.

Katalog 144

Jahr 1900.

In Berlin für 1 Mark versteigert.

\section{Camps y Soler, Oscar}

Estudios filosóficos sobre la música. (La música en el templo, en la sala y en el teatro). Madrid 1864.

Katalog 144.

Jahr 1900.

In Berlin für 3 Mark versteigert.

\section{Cano, Antonio}

Método completo de guitarra con un tratado de armonía aplicado a este instrumento.

Katalog 144.

Jahr 1900.

In Berlin für 8 Mark versteigert.

\section{Eslava, Hilarión}

Misa de difuntos a voces y orquesta. Madrid 1861. Enthält 122 seiten.

Katalog 216.

In Berlin für 24 Mark versteigert.

Escuela de composición. Tratado primero: de la armonía. Madrid, 1861.

Katalog 197.

In Berlin für 12,50 Mark versteigert.

Fondevilla, Antonio

Ofertorio en Sol para órgano. Madrid . 
Katalog 144.

Jahr 1900.

In Berlin für 1,50 Mark versteigert.

\section{Fuertes, Soriano}

Música árabe-española, y conexión de la música con la astronomía, medicina y arquitectura. Barcelona, 1853.

Enthält 133 seiten.

Katalog 100.

Jahr 1893.

In Berlin für 8 Mark versteigert.

Tratado de armonía. Madrid.

Katalog 156.

Jahr 1905.

In Berlin für 3,50 Mark versteigert.

Método breve de Solfeo. Madrid.

Katalog 156.

Jahr 1905

In Berlin für 4 Mark versteigert.

\section{Gimeno, Román}

Fantasía para órgano. Madrid .

Katalog 144.

Jahr 1900.

In Berlin für 1,50 Mark versteigert.

\section{Inzenga, José}

Algunos apuntes sobre el arte de acompañar al piano. Madrid 1870.

Katalog 144.

Jahr 1900.

In Berlin für 1 Mark versteigert.

\section{Iñiguez, Buenaventura}

Método completo de Canto-llano, dedicado a los seminarios conciliares y colegios de misioneros. Madrid 1871.

Katalog 144.

Jahr 1900.

In Berlin für 5 Mark versteigert.

\section{Jiménez, Juan}

Breve tratado del arte mímica aplicada al canto. Madrid 1862.

Katalog 144.
Jahr 1900.

In Berlin für 3 Mark versteigert.

\section{Laguna, José Flores}

Cuadro sinóptico histórico musical. Madrid, 1862.

Katalog 140.

Jahr 1899

In Berlin für 8 Mark versteigert.

\section{Latorre, Juan Castro}

Método de contrabajo aplicable al de tres y al de cuatro cuerdas. Madrid.

Katalog 144.

Jahr 1900.

In Berlin für 4 Mark versteigert.

\section{Martín, Luís García}

Manual de teatros y espectáculos públicos con la reseña histórica y descripción delas salas y circos destinados a ellos. Madrid 1860.

Katalog 144.

Jahr 1900.

In Berlin für 5 Mark versteigert.

\section{Medrano}

Album de la Zarzuela. Madrid 1857.

Katalog 144.

Jahr 1900.

In Berlin für 5 Mark versteigert.

\section{Melcior, $\mathbf{C}$.}

Diccionario enciclopédico de la música. Lérida 1859.

Katalog 144.

Jahr 1900.

In Berlin für 5 Mark versteigert.

\section{Menchaca, Angel}

Mozart y su Don Juan. Buenos Aires, 1889.

Katalog 144.

Jahr 1900

In Berlin für 2,50 Mark versteigert.

\section{Millán, Luis Carmena}

Crónica de la ópera italiana de Madrid desde el año 1738 hasta nuestros días. Madrid 1878.

Katalog 144.

Jahr 1900.

In Berlin für 12 Mark versteigert. 
Miró, José

Método de piano adoptado para la enseñanza de los alumnos de la escuela nacional de música. Madrid. Katalog 144.

Jahr 1900.

In Berlin für 6 Mark versteigert.

\section{Moretti, Federico}

Principios para tocar la guitarra de seis ordenes, precedidos de los elementos generales de la música. Madrid 1807.

Katalog 144.

Jahr 1900.

In Berlin für 20 Mark versteigert.

\section{Navas, Francisco Marcos}

Arte o compendio general de Canto llano figurado y órgano, en método fácil, ilustrado con algunos documentos, divididos en cinco tratados. Madrid 1816. Katalog 144.

Jahr 1900.

In Berlin für 25 Mark versteigert.

\section{Nombela, J.}

Manual de música. Historia-Gramática. París, 1860.

Katalog 144.

Jahr 1900.

In Berlin für 5 Mark versteigert.

\section{Pedrell, Felipe}

Hispanie Schola Sacra.

Katalog 110.

Jahr 1894.

In Berlin für 10 Mark versteigert.

\section{Peña y Goñi, Antonio}

La ópera española y la música dramática en España en el siglo XIX. Madrid, 1881.

Enthält 133 seiten.

Katalog 129.

Jahr 1889.

In Berlin für 8 Mark versteigert.

\section{Romero y Andia, Antonio}

Gramática musical o sea teoría general de la música. Madrid 1871.

Katalog 144.

Jahr 1900.
In Berlin für 5 Mark versteigert.

\section{Romero, Antonio}

Explicación y ejercicos prácticos para el clarinete sistema Romero. Madrid, 1867.

Katalog 140.

Jahr 1899

In Berlin für 8 Mark versteigert.

\section{Saldoni, Basaltar}

Reseña histórica de la escolanía o colegio de la música de la Virgen de Montserrat en Cataluña desde 1456 hasta hoy día. Madrid 1856.

Katalog 144.

Jahr 1900.

In Berlin für 9 Mark versteigert.

\section{Saldoni, Basaltar}

Efemérides de músicos españoles. Madrid 1860.

Katalog 88.

Jahr 1890.

In Berlin für 10 Mark versteigert.

\section{Teoría de la escritura musical}

Teoría de la escritura musical y su interpretación, poesía en verso para su más fácil inteligencia por D. E. L y D. R. T. Madrid 1870.

Katalog 144.

Jahr 1900.

In Berlin für 2,50 Mark versteigert.

\section{Valero, José y Romero, Antonio}

Nuevo método completo de Solfeo. Madrid.

Katalog 144.

Jahr 1900.

In Berlin für 3 Mark versteigert.

\section{$\underline{\text { XX Jh. }}$}

\section{Subirá, José}

La música en la casa de Alba. Estudios históricos y biográficos. Madrid, 1927. Madrid 1860.

Katalog 223.

Jahr 1931.

Enthält 366 seiten.

In Berlin für 86 Mark versteigert. 


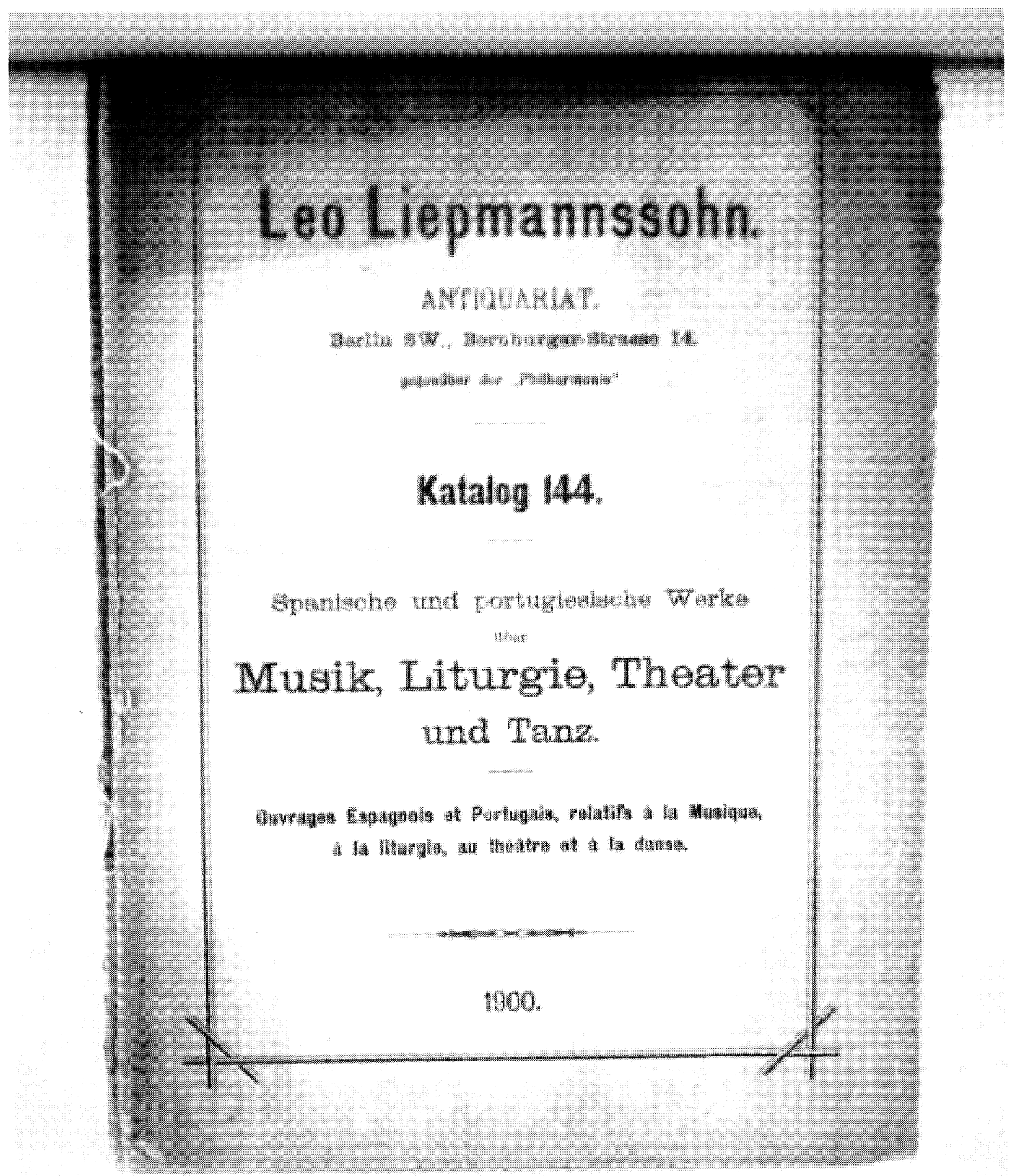

